\title{
Transmission Properties of the Periodic Structures Based on Graphene Nonlinear Optical Conductivity in a Terahertz Field
}

\author{
Reza Ghayoor and Alireza Keshavarz \\ Department of Physics, Shiraz University of Technology, Shiraz, Iran \\ Corresponding author Email: R.Ghayoor@sutech.ac.ir \\ Received: Aug. 23, 2017, Revised: Dec. 24, 2017, Accepted: Jan. 25, 2018, Available Online: June. 30, 2019
}

DOI: 10.29252 /ijop.13.1.35

\begin{abstract}
By developing the terahertz (THz) technology, in addition to generators and detectors of $\mathrm{THz}$ waves, the existence of some tools such as modulators and filters are needed. THz filters are important tools for various applications in the field of chemical and biological sensors. Linear and nonlinear optical properties of the graphene have attracted lots of attention. In fact graphene exhibits various nonlinear phenomena. Hence in this paper, by entering the graphene to the field of $\mathrm{THz}$ and using the graphene nonlinear properties with utilizing the transfer matrix method and transmission properties of a periodic structure containing graphene are investigated. A fairly straightforward computational method allows us to examine the effect of different structural parameters on the transmittance spectrum. Simulation results show that if the graphene nonlinear response in a periodic structure in the presence of a high-intensity $\mathrm{THz}$ field is considered, the proposed structure displays two bands of passes and stopping which can improve the design of the filters and controllers of $\mathrm{THz}$ waves.
\end{abstract}

KEYWORDS: Graphene, Transmittance spectrum, Terahertz filters, Transfer matrix method.

\section{I.INTRODUCTION}

Graphene is a single atomic layer of carbon atoms tightly packed into a highly symmetric two-dimensional honeycomb lattice. The optical properties of graphene have been studied both experimentally and theoretically. In the $\mathrm{THz}$ to the far-infrared (FIR) spectral regime optical conductance of graphene-based systems attracts many researchers' interests due to the researches searched on viable $\mathrm{THz}$ detectors and emitters [1].

The fact that graphene has attracted intensive scientific interest is because of its incredible physical properties showing great potential applications in nano and optoelectronic devices, and it has outstanding optical properties, such as strong light-graphene interaction, broadband and high-speed operation, etc. Some graphene/dielectric heterostructures are also proposed to manipulate $\mathrm{THz}$ radiations. Especially, the electrical tunability of conductivity of graphene can potentially open a new possibility of tunable optical sensor, metamaterials, $\mathrm{THz}$ absorber, and tunable filters, etc. [2].

In addition, linear and nonlinear optical properties of graphene as $2 \mathrm{D}$ materials are very interesting. Graphene is a centrosymmetric material that exhibits large third-harmonic generation (THG), strong optical Kerr nonlinearity, and induced secondorder nonlinearity in a single atomic layer. This allows us to employ graphene in active photonic devices with improved functionality, including ultracompact modulators, optical limiters, frequency converters, and photovoltaic and photo resistive devices. These advantages require advances in nanofabrication and experimental techniques, theoretical models, and numerical methods for modeling the devices incorporating 2D materials [3].

T. Zhan et al. developed a transfer matrix method for optical calculations in non- 
interacting graphene layers. Within the framework of this method, the transfer matrices for various graphene layers which has linear conductivity can be obtained, from which the reflectance, transmittance and absorbance spectra of graphene layers can be easily obtained [4].

Ghasempour et al. derived a transfer matrix for optical calculations in anisotropic graphene layers in the presence of an external magnetic field. Proposed transfer matrix to study surface plasmons in a multilayer structure containing three anisotropic graphene layers which have linear conductivity is applied [5].

Nonlinear optical properties of multilayer graphene can be determined by Z-scan method. The measurements are carried out at $1150,1550,1900$ and $2400 \mathrm{~nm}$ with a 100femtosecond laser source. Under laser illumination, the multi-layer graphene exhibits a transmittance increase due to saturable absorption [6].

On the other hand, Xiao et al. experimentally demonstrated graphene-plasmon polariton excitation in a continuous graphene monolayer resting on a two-dimensional subwavelength silicon grating. These dielectric-graphene series are fabricated based on low-cost nano sphere lithography. An improved method for transferring CVD-graphene on copper is used to obtain a high quality of the transferred graphene monolayer $[7,8]$.

In recent years, $\mathrm{THz}$ technology has been studied very much and the field of production and detection of these waves have been a lot of development. Also, the potential applications of these waves, telecommunications, imaging, security, etc. are mentioned [9].

Filters play an important role in applications such as $\mathrm{THz}$ signal processing, telecommunications, medical and chemical sensors and biosensors. Efforts are done to develop and design high-pass and tunable filters. These filters are usually based on photonic crystals, liquid photonic crystals [10]. $\mathrm{He}$ et al. suggests that the graphene- $\mathrm{SiO}_{2}-\mathrm{Si}$
$\left(\mathrm{GSiO}_{2} \mathrm{Si}\right)$ structure is based on the flexible substrate to realize dynamically control of the propagation waves. Since the permittivity of graphene layer can be varied via the applied electric fields or chemical doping, the transmission of the $\mathrm{GSiO}_{2} \mathrm{Si}$ structure can be conveniently modulated. Therefore, the tunable transmission properties of the proposed $\mathrm{GSiO}_{2} \mathrm{Si}$ structure has been explored in the $\mathrm{THz}$ regimes. They show that transmission of the proposed $\mathrm{GSiO}_{2} \mathrm{Si}$ structure can be modulated conveniently in a broadband range [11].

In this paper, we theoretically examine the transmission properties of a one-dimensional periodic structure including graphene in two stages using the transfer matrix method. In this way, first we consider the graphene only with linear conductivity (intraband conductivity) and then in addition to linear conductivity, the third order conductivity is also considered. Our main goal in this study is to utilize nonlinear graphene and changes of the structural parameters in order to design tunable filters in the range of terahertz.

\section{THEORETICAL MODEL AND METHOD}

Here, we introduce the transfer matrix method to calculate the transmission coefficients at the interface of graphene-dielectric. The main idea of the transfer matrix is that the electric and magnetic fields of different places can be linked to each other. We also examine the propagation of transverse magnetic (TM) polarized waves in the graphene layer. Figure 1 shows the structure of a unit cell of the dielectric-graphene.

In this structure, the dielectric layer thickness is $d$ and the thin layer graphene has the conductivity $\sigma_{g}$. The graphene-dielectric interface matrix will consider as $M$ that can link the field of the layer $n$ to the layer $n+1$ [12]:

$M=\left[\begin{array}{cc}(2+i \kappa \xi) / 2 u & -i \kappa \xi / 2 \\ i \kappa \xi / 2 & (2-i \kappa \xi) u / 2\end{array}\right]$ 
where parameters $\xi, \kappa$, and $u$ are defined as follows:

$\xi=\frac{\sigma_{g} \eta_{0}}{i k_{0}}$

$\kappa=k_{0} \cos \theta$

$u=\exp (i k d)$

where $\eta_{0}$ is air impedance, $k_{0}=\frac{2 \pi}{\lambda}$, and $\theta$ is angle of incident beam to the structure.

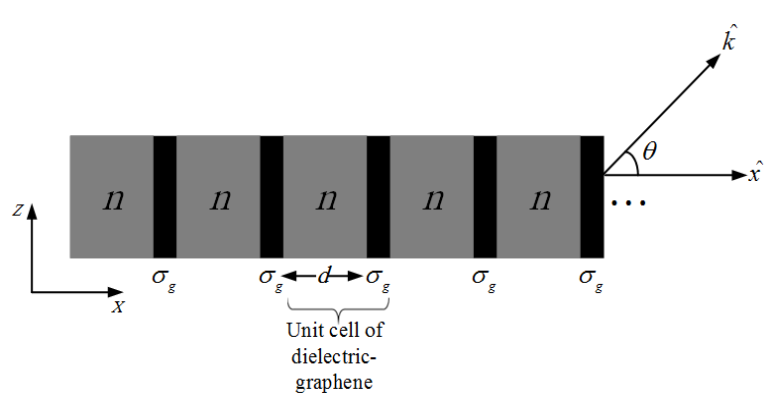

Fig. 1. One dimensional unit cell of the proposed structure containing dielectric layer with a refractive index of $n$ and thickness of $d$, and graphene layer with a total conductivity of $\sigma_{g}$ under radiation of wave with angular frequency $\omega$ and incident angle $\theta$.

Without considering the external magnetic field and under the random-phase approximation, the isotropic surface conductivity $\sigma$ of graphene in the $\mathrm{THz}$ frequencies is dominated by the $\mathrm{THz}$ transitions. In the limit $\omega \tau$ ? 1 , the linear part of the surface conductivity can be expressed as [13]:

$\sigma_{\text {intra }}(\omega)=\frac{i e^{2} \mu_{c}}{\pi \mathrm{h}^{2}\left(\omega+i \tau^{-1}\right)}$

where $\mathrm{h}$ is reduced Planck's constant, $e$ is electron charge, $\mu_{c}$ is chemical potential of graphene, $\tau$ is relaxation time of charge carriers.

This model is applicable in low-temperature limit $\left(K_{B} T=\mu_{c}\right)$ at low frequency $\left(\mathrm{h} \omega \leq \mu_{c}\right)$.
For the strong field condition, the nonlinear part of the conductivity must be considered [14]:

$\sigma_{g}=\sigma_{L}+\sigma^{N L}\left|E_{\tau}\right|^{2}$

and the total conductivity of graphene reads where $E_{\tau}$ is the tangential component of the electric field that is considered as $E_{\tau}=E_{0} \exp \left(-i k_{0} \sqrt{\varepsilon} \cos \theta\right) . \quad \sigma_{N L}$ denotes the third order nonlinear conductivity that for the $\mathrm{THz}$ regime can be acquired from Mikhailov and Ziegler [15]:

$\sigma_{3, T H z}=-i \frac{3 e^{4} v_{f}^{2}}{32 \omega^{3} \mathrm{~h} \mu_{c}}$

where $v_{f}=10^{6} \mathrm{~m} / \mathrm{s}$ is the Fermi velocity of electrons.

If the unit cell structure of Fig. 1 is repeated periodically for a number of periods, such as $N$, the entire matrix is considered as follows:

$U=\prod_{n=1}^{N} M_{n}$

After obtaining the matrix $U$, the reflection and transmission coefficients can be obtained from the elements of the matrix as [12]:

$$
\begin{aligned}
& r=\frac{U(2,1)}{U(1,1)} \\
& t=\frac{1}{U(1,1)}
\end{aligned}
$$

\section{SimULATION}

In this section, we examine the influence of structural parameters on the transmittance spectrum of Fig 1.

\section{A. Effect of the dielectric layer}

Transmittance spectrum diagrams for two thicknesses $d=0.75 \mu \mathrm{m}$ and $d=1.5 \mu \mathrm{m}$ are simulated and the results are presented in Fig. 2. Simulations for a range of 0.3 to 100 $\mathrm{THz}$ are plotted for two cases. The first case 
involves only linear conductivity (intraband conductivity), and the second case, which includes total conductivity that including linear and third order conductivity $\left(\sigma_{3, T H z}\right)$.

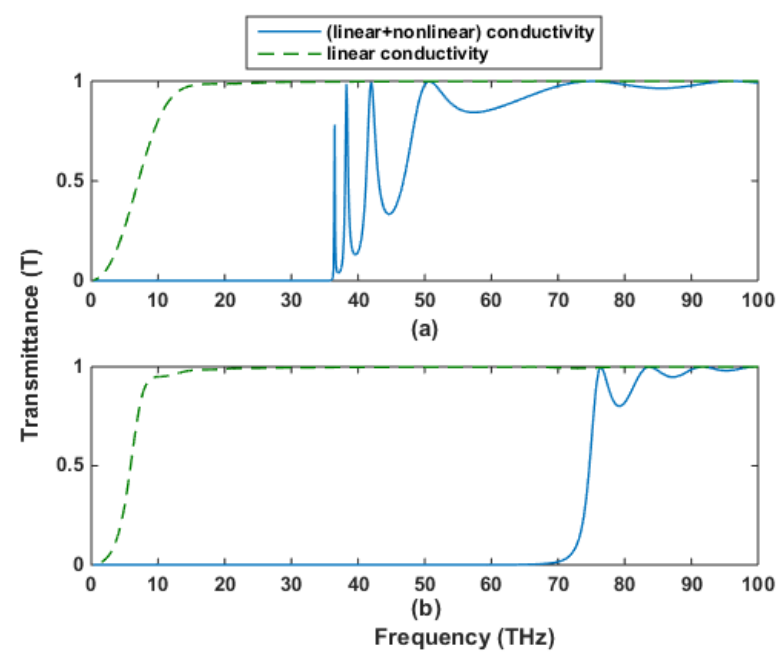

Fig. 2. Transmittance spectra for linear conductivity and total conductivity of graphene (summation of linear conductivity and nonlinear conductivity). $n=1.46, \quad N=10, \quad \theta=\pi / 6, \quad \tau=0.5 T H z$, $\mu_{c}=0.2 \mathrm{eV}$. (a) for $d=0.75 \mu \mathrm{m} \quad$ (b) for $d=1.5 \mu \mathrm{m}$.

The high intensity of the amplitude of the incident wave field leads to the nonlinearity being dominant. In this structure by increasing the thickness of the dielectric layer, in case that graphene in the structure has total conductivity (linear+nonlinear), the stop band of the transmittance spectrum increases and also reduces the number of frequency peaks. In the other words, by increasing the thickness of the dielectric layer, this structure acts in such a way that it prevents the transmission of further frequencies, and subsequently passes fewer frequencies, on the other hand, the high frequency peaks that are seen in the center frequencies of Fig. 3(a), are eliminated.

\section{B. Effect of the chemical potential of graphene}

The chemical potential of graphene in the periodic structure of Fig. 1 plays an important role in the variation of the transmittance and spectrum of this structure. The chemical potential of graphene usually is changed by applying an electric field or by doping the elements within the graphene lattice. Figure 3 shows the simulation results for both values $\mu_{c}=0.1 \mathrm{eV}$ and $\mu_{c}=0.3 \mathrm{eV}$.

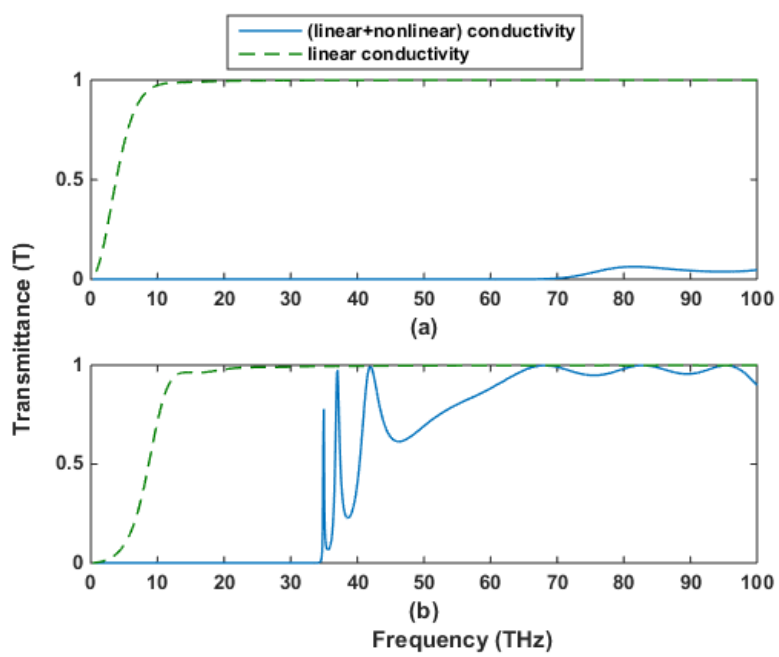

Fig. 3. Transmittance spectra for linear conductivity and total conductivity of graphene (summation of linear conductivity and nonlinear conductivity). $n=1.46, \quad N=10, \quad \theta=\pi / 6, \quad \tau=0.5 T H z$, $d=1 \mu \mathrm{m}$. (a) for $\mu_{c}=0.1 \mathrm{eV}$ (b) for $\mu_{c}=0.3 \mathrm{eV}$.

As shown in Fig. 3(a) and 3(b), when the nonlinear effect of graphene is considered, increasing in the chemical potential reduces the stop band of transmittance spectrum and leads to the oscillation of it at high $\mathrm{THz}$ frequency, while more frequency peaks are detected. The simulation results of Fig. 3(a) and 3(b) are performed for frequencies of more than $100 \mathrm{THz}$ and forned that the transmittance spectrum reaches a maximum constant value. In the central frequencies of this spectrum, there are sharp frequency peaks that improve the detection of this frequency range, and as Fig. 3(b) shows, with increasing the number of graphene layers, these frequency peaks in this range increase. For example, the result of Fig. 3(a) is suitable for designing a filter that is used only for passing a single frequency of about $80 \mathrm{THz}$.

\section{Effect of the angle of incident wave}

Another important parameter in determining the transmittance spectrum of the structure of Fig. 1 is the angle of incident wave incident the structure. Simulations are performed for 
both values of $\theta=22.5^{\circ}$ and $\theta=45^{\circ}$, and the results are presented in Fig. 4.

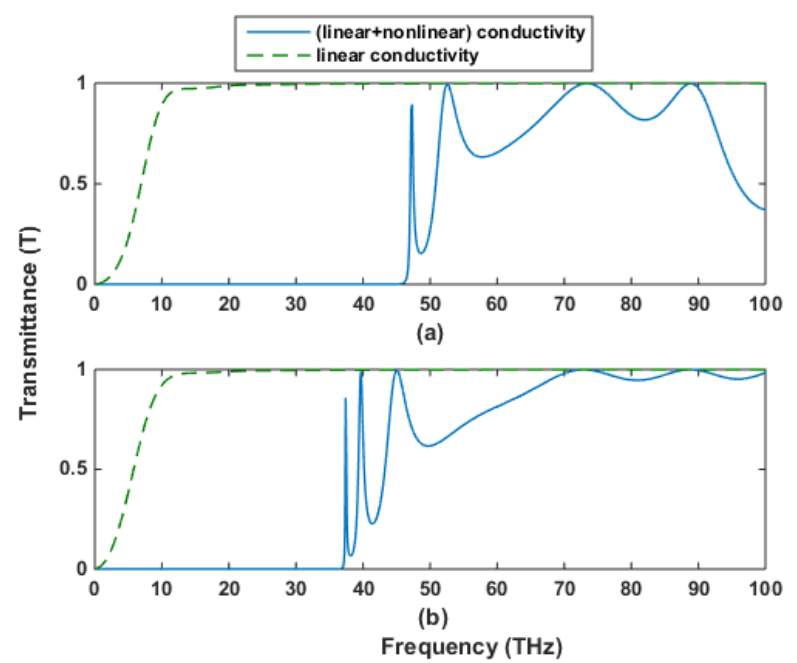

Fig. 4. Transmittance spectra for linear conductivity and total conductivity of graphene (summation of linear conductivity and nonlinear conductivity). $n=1.46, \quad N=10, \quad \mu_{c}=0.2 \mathrm{eV}, \quad \tau=0.5 T H z$, $d=1 \mu \mathrm{m}$. (a) for $\theta=\pi / 4$ (b) for $\theta=\pi / 8$.

Taking into account the total conductivity containing the linear and third order conductivity. Results show that by decreasing the incident wave angle, the stop band in the transmittance spectrum increases and the number of frequency peaks decreases. As shown in Figs. 4(a) and 4(b), by decreasing the angle from $\theta=22.5^{\circ}$ to $\theta=45^{\circ}$, the number of sharp frequency peaks seen in the central frequencies decreases, which means that by decreasing the angle, fewer frequencies through this structure is allowed to pass. If only linear effects of graphene are taken into account, as shown in Fig. 4, most frequencies pass and the system act almost as a transparent environment for the incident wave.

\section{Effect of the dielectric coefficient}

The influence of the dielectric coefficient of materials in periodic structures on the variation of the transmittance spectrum can not be ignored. Simulation results are shown in Fig. 5. According to the third-order conductivity in the calculations, the increase in the dielectric coefficient from $\varepsilon=2.13$ to $\varepsilon=4$, increases the stop band and reduces the number of frequency peaks.

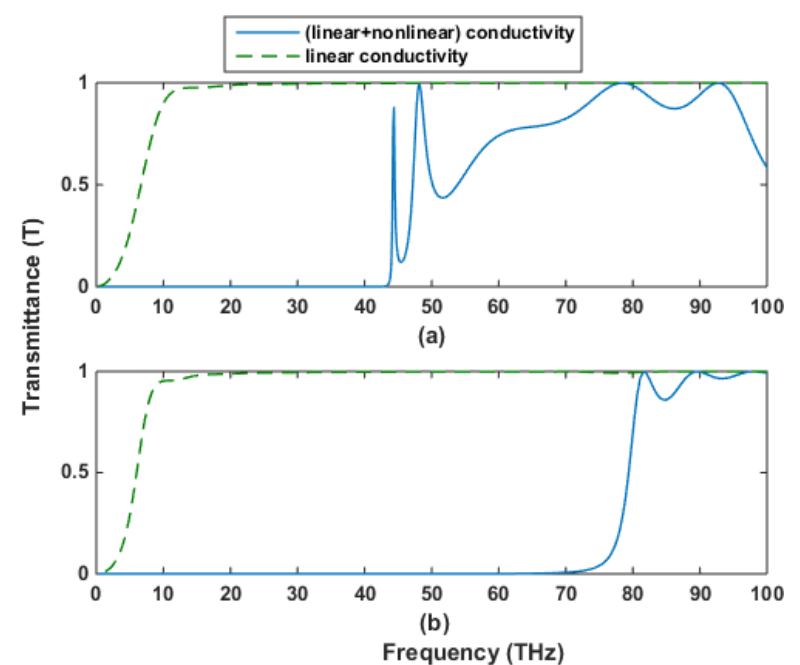

Fig. 5. Transmittance spectra for linear conductivity and total conductivity (summation of linear conductivity and nonlinear conductivity). $d=1 \mu \mathrm{m}$, $N=10, \theta=\pi / 6, \tau=0.5 \mathrm{THz}, \mu_{c}=0.2 \mathrm{eV}$. (a) for $\varepsilon=2.13(\mathrm{n}=1.46)$ (b) for $\varepsilon=4(n=2)$.

In the other words, the non-conductivity of the material between the graphene layers and the high intensity of the $\mathrm{THz}$ field make the structure of Fig. 1, like a strong reflector, which returns most of the low frequencies. Although the increase in the dielectric coefficient removes the transmission spectrum from the oscillatory state for the end of the frequency range and generates frequency peaks in this range, this increase in the dielectric coefficient also eliminates the number of frequency peaks seen in the central frequencies.

In Figs. 2-5, we can see dips in the transmittance spectra. For example, in Fig. 5(b), there are dips at 85 and $92 \mathrm{THz}$ frequencies. These dips can be associated with the generation of plasmonic waves. As it can be seen, by changing the structural parameters it is possible to control the increase or decrease of the plasmonic waves produced in this structure. We can attribute the generation of these plasmonic waves to the presence of graphene with nonlinear conductivity in the structure of Fig. 1.

\section{IV.CONCLUSION}

In this paper, we examine the transmission properties of the periodic systems contain of 
graphene, taking into account the third order conductivity of graphene in the presence of high intensity $\mathrm{THz}$ field. It is shown by the simulation results for the proposed structure, changing the structural parameters such as the dielectric layer thickness, the graphene chemical potential, the variation in the incident wave angle and the change in the dielectric coefficient, the transmission and the reflection spectrum of the system can be changed. These results can be used to design the bandpass filters and the controllers of $\mathrm{THz}$ waves. Changing structural parameters leads to improve the design of adjustable $\mathrm{THz}$ filters.

\section{REFERENCES}

[1] J. Lv, R.Y. Yuan, and H. Yan, "Temperaturedependent of nonlinear optical conductance of graphene-based systems in high-intensity terahertz field," Nano-Micro Lett. Vol. 6, pp. 153-162, 2014.

[2] X. Dai, L. Jiang, and Y. Xiang, "Tunable optical bistability of dielectric/nonlinear graphene/dielectric heterostructures," Opt. Express, Vol. 23, pp. 6497-6508, 2015.

[3] M. Weismann, and N.C. Panoiu, "Theoretical and computational analysis of second-and third-harmonic generation in periodically patterned graphene and transition-metal dichalcogenide monolayers," Phys. Rev. B, Vol. 94, pp. 035435 (1-28), 2016.

[4] T. Zhan, X. Shi, Y. Dai, X. Liu, and J. Zi, "Transfer matrix method for optics in graphene layers," J. Phys. Condens. Matter, Vol. 25, pp. 215301 (1-9), 2013.

[5] A.G. Ardakani, Z. Ghasemi, and M.M. Golshan, "A new transfer matrix for investigation of surface plasmon modes in multilayer structures containing anisotropic graphene layers," Eur. Phys. J, Vol. 132, pp. 206-218, 2017.

[6] G. Demetriou, H.T Bookey, F. Biancalana, E. Abraham, Y. Wang, and A.K. Kar, "Nonlinear optical properties of multilayer graphene in the infrared," Opt. Express, Vol. 24, pp. 13033-13043, 2016.

[7] X. Zhu, W. Yan, P. Uhd Jepsen, O. Hansen, N. Asger Mortensen and S. Xiao, "Experimental observation of plasmons in a graphene monolayer resting on a twodimensional subwavelength silicon grating,"
Appl. Phys. Lett., Vol. 102, pp. 131101 (112), 2013.

[8] K. Tantiwanichapan, X. Wang, H. Durmaz, Y. Li, A.K. Swan, and R. Paiella, "Graphene Terahertz Plasmons: A Combined Transmission Spectroscopy and Raman Microscopy Study," ACS Photon. Vol. 4, pp. 2011-2017, 2017.

[9] N. Vieweg, N. Born, I. AL.Naib, and M. Koch "Electrically tunable terahertz notch filters," J. Infrared Millim. Terahertz, Vol. 33, pp. 327332, 2012.

[10] Z. Zhu, X. Zhang, J. Gu, R. Singh, Z. Tian, J. Han, and W. Zhang, "A metamaterial-based terahertz low-pass filter with low insertion loss and sharp rejection", IEEE Trans. Terahertz Sci. Vol. 3, pp. 832-837, 2013.

[11] X. He, "Tunable terahertz graphene metamaterials," Carbon, Vol. 82, pp. 229-237, 2015.

[12] B. Wang, X. Zhang, K. Ping Loh, and J. Teng, "Tunable broadband transmission and phase modulation of light through grapheme multilayers," Appl. Phys. Vol. 115, pp. 213102 (1-8), 2014.

[13] X. Dai, L. Jiang, and Y. Xiang, "Tunable $\mathrm{THz}$ angular/frequency filters in the modified Kretschmann-Raether configuration with the insertion of single layer graphene," IEEE Photon. J. Vol. 7, pp. 1-8, 2015.

[14] X. Jiang, J. Bao, B. Zhang and X. Sun, "Dual nonlinearity controlling of mode and dispersion properties in graphene-dielectric plasmonic waveguide," Nanoscale Res. Lett. Vol. 12, pp. 395-401, 2017.

[15] A. Pitilakis, D. Chatzidimitriou, and E.E. Kriezis, "Theoretical and numerical modeling of linear and nonlinear propagation in graphene waveguides," Opt. Quantum Electron. Vol. 48, pp. 243-264, 2016. 


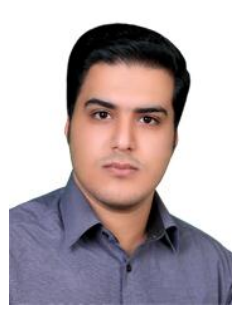

Reza Ghayoor was born in Isfahan, Iran. He received his B.Sc. degree in Optics and laser engineering from Maleke Ashtar University of Technology in 2015, M.Sc. degree in Photonic from Shiraz University of Technology in 2018. His master thesis was focused on solar cells with the subject of "Fabrication and Characterization of Dye Sensitized Solar Cell Containing Reduced Graphene Oxides". He is the author of more than 5 papers. He has worked on much scientific research area such as Dye sensitized solar cells, Terahertz waves, Nano- Photonics and Nano-Physics (Graphene and Quantum Dots). At the present, he is actively involved in optics and photonics.

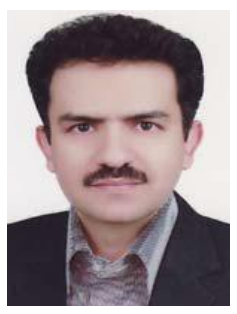

Alireza Keshavarz was born in Shiraz, Iran. $\mathrm{He}$ received his B.Sc. degree in Atomic Physics from Shiraz University in 1995, M.Sc. degree in Atomic and molecular Physics from Kerman University in 1997, and the Ph.D. degree in Physics (laser and optics) from Shiraz University in 2004. He has worked on much scientific research area such as Nonlinear Optics and Laser, Optical Solitons, Nano- Photonics and Nano-Physics (Quantum Dots and Wires). He joined the Department of Physics of Shiraz University of Technology in 2004. He promoted to Associate Professor in 2012 and Professor in 2016. At the present, he is actively involved in optics and photonics and supervises M.Sc. and Ph.D. students in these fields. 
THIS PAGE IS INTENTIONALLY LEFT BLANK. 